\title{
Nuclear Lattice Simulations with Chiral Effective Field Theory
}

\section{Dean Lee*}

Department of Physics, North Carolina State University, Raleigh, NC 27695, USA

E-mail: dean_lee@ncsu.edu

We present recent results on lattice simulations using chiral effective field theory. In particular we discuss lattice simulations for dilute neutron matter at next-to-leading order and three-body forces in light nuclei at next-to-next-to-leading order.

8th Conference Quark Confinement and the Hadron Spectrum

September 1-6 2008

Mainz, Germany

* Speaker. 
Lattice simulations based on the framework of effective field theory have been used in studies of nuclear matter [1] and neutron matter [2, 3, 4]. The method has also been used to study light nuclei in pionless effective field theory [5] and chiral effective field theory at leading order (LO) [6]. More recently next-to-leading order (NLO) calculations have been carried out for the ground state of neutron matter [7,8]. A review of lattice effective field theory calculations can be found in Ref. [9]. In this proceedings article we describe some new results which, at the time of this writing, are not yet published.

At leading order in chiral effective field theory the nucleon-nucleon effective potential is

$$
V_{\mathrm{LO}}=V+V_{I^{2}}+V^{\mathrm{OPEP}} \text {. }
$$

$V, V_{I^{2}}$ are the two independent contact interactions at leading order in the Weinberg power counting scheme, and $V^{\mathrm{OPEP}}$ is the instantaneous one-pion exchange potential. The interactions in $V_{\mathrm{LO}}$ can be described in terms of their matrix elements with two-nucleon incoming and outgoing momentum states. For bookkeeping purposes we label the amplitude as though the two interacting nucleons were distinguishable, $A$ and $B$. In the following $\vec{q}$ denotes the $t$-channel momentum transfer while $\vec{k}$ is the $u$-channel exchanged momentum transfer. We use $\tau_{I}$ with $I=1,2,3$ to represent Pauli matrices acting in isospin space and $\sigma_{S}$ with $S=1,2,3$ to represent Pauli matrices acting in spin space.

For the two leading-order contact interactions the amplitudes are

$$
\begin{gathered}
\mathscr{A}(V)=C, \\
\mathscr{A}\left(V_{I^{2}}\right)=C_{I^{2}} \sum_{I} \tau_{I}^{A} \tau_{I}^{B} .
\end{gathered}
$$

For the one-pion exchange potential,

$$
\mathscr{A}\left(V^{\mathrm{OPEP}}\right)=-\left(\frac{g_{A}}{2 f_{\pi}}\right)^{2} \frac{\sum_{I} \tau_{I}^{A} \tau_{I}^{B} \sum_{S} q_{S} \sigma_{S}^{A} \sum_{S^{\prime}} q_{S^{\prime}} \sigma_{S^{\prime}}^{B}}{q^{2}+m_{\pi}^{2}} .
$$

For our physical constants we take $m=938.92 \mathrm{MeV}$ as the nucleon mass, $m_{\pi}=138.08 \mathrm{MeV}$ as the pion mass, $f_{\pi}=93 \mathrm{MeV}$ as the pion decay constant, and $g_{A}=1.26$ as the nucleon axial charge.

In Ref. [6] two different lattice actions were considered which were later denoted $\mathrm{LO}_{1}$ and $\mathrm{LO}_{2}$ [7]. The interactions in $V_{\mathrm{LO}_{1}}$ include one-pion exchange and two zero-range contact interactions corresponding with amplitude

$$
\mathscr{A}\left(V_{\mathrm{LO}_{1}}\right)=C+C_{I^{2}} \sum_{I} \tau_{I}^{A} \tau_{I}^{B}+\mathscr{A}\left(V^{\mathrm{OPEP}}\right) .
$$

The interactions in $V_{\mathrm{LO}_{2}}$ consist of one-pion exchange and two Gaussian-smeared contact interactions,

$$
\mathscr{A}\left(V_{\mathrm{LO}_{2}}\right)=C f(\vec{q})+C_{I^{2}} f(\vec{q}) \sum_{I} \tau_{I}^{A} \tau_{I}^{B}+\mathscr{A}\left(V^{\mathrm{OPEP}}\right),
$$

where $f(\vec{q})$ is a lattice approximation to a Gaussian function. The smeared interactions in $\mathrm{LO}_{2}$ are used to better reproduce $S$-wave phase shifts for nucleon momenta up to the pion mass. The coefficients $C$ and $C_{I^{2}}$ are tuned to reproduce the physical $S$-wave scattering lengths. In Ref. [7] 
nucleon-nucleon phase shifts were calculated for these two lattice actions using the spherical wall method [10] at spatial lattice spacing $a=(100 \mathrm{MeV})^{-1}$ and temporal lattice spacing $a_{t}=(70$ $\mathrm{MeV})^{-1}$. For each case NLO corrections were also computed perturbatively and the unknown operator coefficients determined by fitting to low-energy scattering data.

In Ref. [8] the ground state energy for dilute neutron matter was computed using the lattice action $\mathrm{LO}_{2}$ and auxiliary-field Monte Carlo. Next-to-leading-order corrections to the energy were also calculated perturbatively. In this calculation the largest source of systematic error was the large size of NLO corrections for Fermi momenta larger than $100 \mathrm{MeV}$. This was due to attractive $P$-wave interactions generated by Gaussian smearing in $\mathrm{LO}_{2}$ that needed to be cancelled at nextto-leading order. In systems with both protons and neutrons this $P$-wave correction is numerically small when compared with the strong binding produced by $S$-wave interactions. For pure neutron matter, however, the $S$-wave interactions produce much less binding due to Fermi repulsion. Therefore on a relative scale, the $P$-wave interactions are not as small an effect in neutron matter.

These problems have been resolved using a new leading-order action $\mathrm{LO}_{3}$ [20]. The interactions in $V_{\mathrm{LO}_{3}}$ correspond with the amplitude,

$$
\begin{aligned}
\mathscr{A}\left(V_{\mathrm{LO}_{3}}\right) & =C_{S=0, I=1} f(\vec{q})\left(\frac{1}{4}-\frac{1}{4} \sum_{S} \sigma_{S}^{A} \sigma_{S}^{B}\right)\left(\frac{3}{4}+\frac{1}{4} \sum_{I} \tau_{I}^{A} \tau_{I}^{B}\right) \\
& +C_{S=1, I=0} f(\vec{q})\left(\frac{3}{4}+\frac{1}{4} \sum_{S} \sigma_{S}^{A} \sigma_{S}^{B}\right)\left(\frac{1}{4}-\frac{1}{4} \sum_{I} \tau_{I}^{A} \tau_{I}^{B}\right)+\mathscr{A}\left(V^{\mathrm{OPEP}}\right) .
\end{aligned}
$$

The Gaussian-smeared interactions are multiplied by spin and isospin projection operators. Only the $C_{S=0, I=1}$ term contributes in pure neutron matter. Using the $\mathrm{LO}_{3}$ action with NLO corrections, we have computed the ground state energy for dilute neutrons in a periodic box [20]. For spatial lattice spacing $a=(100 \mathrm{MeV})^{-1}$ and temporal lattice spacing $a_{t}=(70 \mathrm{MeV})^{-1}$ simulations were done with 8, 12, 16 neutrons in periodic boxes with lengths $L=4,5,6,7$. In Fig. 1 we show results for the ratio of the interacting ground state energy to non-interacting ground state energy, $E_{0} / E_{0}^{\text {free }}$, as a function of Fermi momentum $k_{F}$. For comparison we show other results from the literature: FP 1981 [11], APR 1998 [12], CMPR $v 6$ and $v 8^{\prime}$ [13], SP 2005 [14], GC 2007 [15], and GIFPS 2008 [16].

At next-to-next-to-leading order (NNLO) in chiral effective field theory we find contributions due to three-nucleon forces. These interactions consist of a pure contact interaction, one-pion exchange, and two-pion exchange [17]. The coupling of one or more pions to a single nucleon is constrained by chiral symmetry and the corresponding low energy constants are known [18]. In the limit of exact isospin symmetry there are only two unknown coefficients, one for the three-nucleon contact interaction and one for the two-nucleon-pion vertex involved in the one-pion exchange interaction. At fixed lattice spacing we have determined these two unknown coefficients by fitting to the triton binding energy and spin-doublet nucleon-deuteron scattering phase shifts via Lüscher's finite volume formula [19]. Results for the doublet nucleon-deuteron scattering phase shift are shown in Fig. 2 using the $\mathrm{LO}_{2}$ lattice action for lattice spacing $a=(100 \mathrm{MeV})^{-1}$ and temporal lattice spacing $a_{t}=(150 \mathrm{MeV})^{-1}[20]$.

Having determined the NNLO three-body forces, we have computed the ground state of the alpha particle without Coulomb interactions on a periodic lattice using auxiliary-field projection 


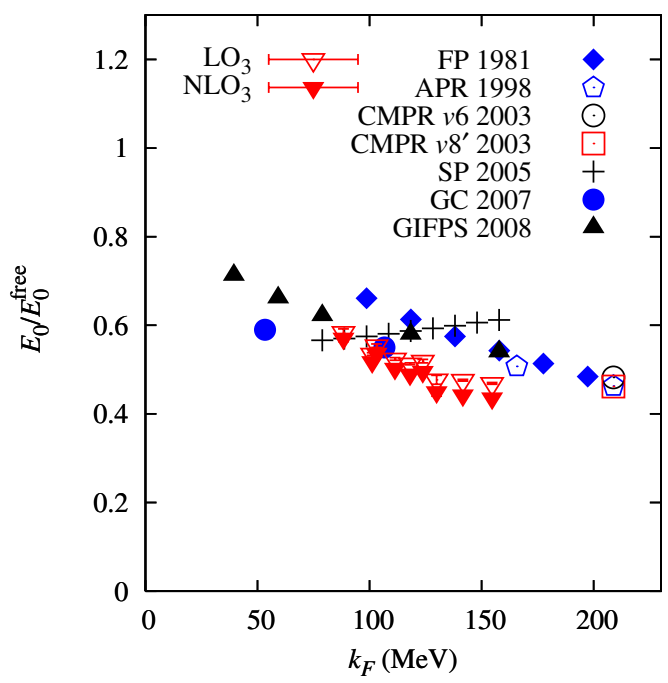

Figure 1: Ground state energy ratio $E_{0} / E_{0}^{\text {free }}$ for $\mathrm{LO}_{3}$ and $\mathrm{NLO}_{3}$ versus Fermi momentum $k_{F}$. For comparison we show results for FP 1981 [11], APR 1998 [12], CMPR $v 6$ and $v 8^{\prime} 2003$ [13], SP 2005 [14], GC 2007 [15], and GIFPS 2008 [16].

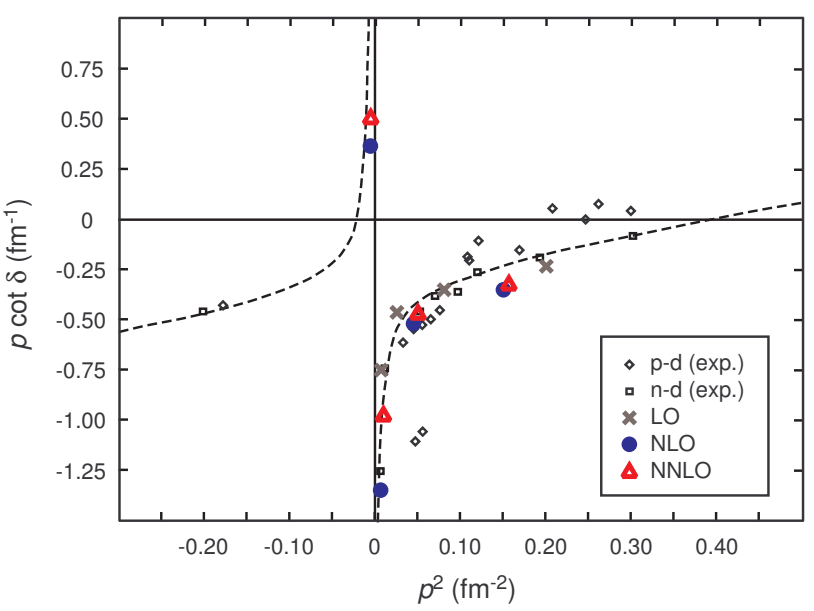

Figure 2: Results for the spin-doublet nucleon-deuteron scattering phase shift at LO, NLO, and NNLO.

Monte Carlo [20]. The NNLO results are within 5\% of the actual Coulomb-subtracted alpha binding energy of about $29 \mathrm{MeV}$. This is consistent with the expected size of errors for our chosen lattice spacing and order in effective field theory.

\section{Acknowledgements}

The work presented here was done in collaboration with Bugra Borasoy, Evgeny Epelbaum, Hermann Krebs, and Ulf-G. Meißner. Partial financial support from the Deutsche Forschungsge- 
meinschaft (SFB/TR 16), Helmholtz Association (contract number VH-NG-222 and VH-VI-231), and U.S. Department of Energy (DE-FG02-03ER41260) are acknowledged. This research is part of the EU Integrated Infrastructure Initiative in Hadron Physics under contract number RII3-CT2004-506078. The computational resources for this project were provided by the Jülich Supercomputing Centre at the Forschungszentrum Jülich.

\section{References}

[1] H.M. Müller, S.E. Koonin, R. Seki, U. van Kolck, Phys. Rev. C61 (2000) 044320 [nucl-th/9910038].

[2] D. Lee, T. Schäfer, Phys. Rev. C72 (2005) 024006 [nucl-th / 0412002 ]; Phys. Rev. C73 (2006) 015201 [nucl-th/ 050 9017]; Phys. Rev. C73 (2006) 015202 [nucl-th/ 0509018 ].

[3] D. Lee, B. Borasoy, T. Schäfer, Phys. Rev. C70 (2004) 014007 [nucl-th / 0402072 ].

[4] T. Abe, R. Seki, [arXiv:0708.2523 [nucl-th]]; [arXiv:0708.2524 [nucl-th]].

[5] B. Borasoy, H. Krebs, D. Lee, U.-G. Meißner, Nucl. Phys. A768 (2006) 179 [nucl-th/ 0510047 ].

[6] B. Borasoy, E. Epelbaum, H. Krebs, D. Lee, U.-G. Meißner, Eur. Phys. J. A31 (2007) 105 [nucl-th/0611087].

[7] B. Borasoy, E. Epelbaum, H. Krebs, D. Lee, U.-G. Meißner, Eur. Phys. J. A35 (2008) 343 [arXiv:0712.2990 [nucl-th]].

[8] B. Borasoy, E. Epelbaum, H. Krebs, D. Lee, U.-G. Meißner, Eur. Phys. J. A35 (2008) 357 [arXiv:0712.2993 [nucl-th]].

[9] D. Lee, [arXiv:0804.3501 [nucl-th]].

[10] B. Borasoy, E. Epelbaum, H. Krebs, D. Lee, U.-G. Meißner, Eur. Phys. J. A34 (2007) 185 [arXiv:0708.1780 [nucl-th]].

[11] B. Friedman, V.R. Pandharipande, Nucl. Phys. A361 (1981) 502.

[12] A. Akmal, V.R. Pandharipande, D.G. Ravenhall, Phys. Rev. C58 (1998) 1804 [nucl-th/ 9804027 ].

[13] J. Carlson, J. Morales, Jr., V.R. Pandharipande, D.G. Ravenhall, Phys. Rev. C68 (2003) 025802 [nucl-th/0302041].

[14] A. Schwenk, C.J. Pethick, Phys. Rev. Lett. 95 (2005) 160401 [nucl-th/ 0506042 ].

[15] A. Gezerlis, J. Carlson, Phys. Rev. C77 (2008) 032801 [axXiv:0711.3006 [nucl-th] ].

[16] S. Gandolfi, A.Y. Illarionov, S. Fantoni, F. Pederiva, K.E. Schmidt, Phys. Rev. Lett. 101 (2008) 132501 [arXiv: 0805.2513 [nucl-th]].

[17] E. Epelbaum, A. Nogga, W. Glöckle, H. Kamada, U.-G. Meißner, H. Witala, Phys. Rev. C66 (2002) 064001 [nucl-th/0208023].

[18] V. Bernard, N. Kaiser, U.-G. Meißner, Int. J. Mod. Phys. E4 (1995) 193 [hep-ph/ 9501384 ].

[19] M. Lüscher, Commun. Math. Phys. 104 (1986) 177; Commun. Math. Phys. 105 (1986) 153; Nucl. Phys. B354 (1991) 531.

[20] E. Epelbaum, H. Krebs, D. Lee, U.-G. Meißner, work in progress. 